DESY 03-071

HU-EP-03/34

SFB/CPP-03-11

\title{
Simulating the Schrödinger functional with two pseudo-fermions
}

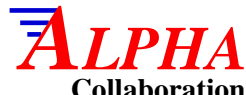 \\ Michele Della Morte ${ }^{a}$, Francesco Knechtli ${ }^{b}$, Juri Rolf, ${ }^{b}$ \\ Rainer Sommer ${ }^{a}$, Ines Wetzorke ${ }^{c}$, Ulli Wolff ${ }^{b}$ \\ ${ }^{a}$ DESY, Platanenallee 6, 15738 Zeuthen, Germany \\ ${ }^{b}$ Institut für Physik, Humboldt Universität, \\ Newtonstr. 15, 12489 Berlin, Germany \\ ${ }^{c}$ NIC/DESY-Zeuthen, \\ Platanenallee 6, 15738 Zeuthen, Germany
}

November 21, 2018

\begin{abstract}
We report on simulations with two flavors of $\mathrm{O}(a)$ improved degenerate Wilson fermions with Schrödinger functional boundary conditions. The algorithm which is used is Hybrid Monte Carlo with two pseudo-fermion fields as proposed by M. Hasenbusch. We investigate the numerical precision and sensitivity to reversibility violations of this algorithm. A gain of a factor two in CPU cost is reached compared with one pseudo-fermion field due to the larger possible step-size.
\end{abstract}




\section{Introduction}

Algorithmic tests with dynamical fermions are large scale projects by themselves. It is therefore a good idea to integrate them in large scale physics projects, part of whose runs are dedicated to study variants of the algorithm or whose configurations are stored and used to further analyze the algorithm. In this work we present a study of a variant of the HMC algorithm which uses two pseudo-fermion fields per degenerate flavor doublet, as proposed by M. Hasenbusch [1,2] and recently tested in [3]. Our simulations of full QCD are performed within the Schrödinger functional framework and deal with two flavors of massless quarks. The Wilson plaquette action is taken for the gauge field and the $\mathrm{O}(a)$ improved Wilson-Dirac operator for the fermions.

The algorithmic study presented here is integrated in the ALPHA project for the computation of the running of the renormalized quark mass [4]. Since we use a finite size technique our lattices range from a very small physical size of $10^{-2} \mathrm{fm}$ to an intermediate size of $1 \mathrm{fm}$. For these lattice volumes we can study the scaling behavior of the HMC algorithm since we can simulate different lattice spacings at fixed physical lattice size.

In Section 2 we describe the main steps to build the HMC algorithm with two pseudo-fermion fields. We explain our choice of the factorization of the Dirac operator into two parts. Section 3 is dedicated to the study of the required numerical precision and to the reversibility of the integration of the molecular dynamics equations of motion. A comparison with HMC using one pseudo-fermion

field is done for several physical lattice sizes and shows that we gain about a factor two in performance when using two pseudo-fermion fields.

\section{HMC with two pseudo-fermion fields}

We would like to simulate on the lattice two flavors of Wilson quarks in the framework of the Schrödinger functional with $\mathrm{O}(a)$ improvement [5]. The Hamiltonian for the evolution in fictitious time is defined as

$$
H=\frac{1}{2} \sum_{x, \mu} \operatorname{Tr}\left[\pi(x, \mu)^{2}\right]+S_{\text {eff }}
$$

where $\pi(x, \mu)$ are the traceless Hermitian momenta conjugate to the gauge field link variables $U(x, \mu)$ and the effective action (at first for one pseudo-fermion field) is given by

$$
S_{\text {eff }}=S_{\mathrm{g}}(U)+S_{\mathrm{det}}(U)+S_{\mathrm{PF}}\left(U, \phi, \phi^{\dagger}\right) .
$$


In eq. (2.2) $S_{g}(U)$ is the Wilson plaquette gauge action with gauge coupling $g_{0}^{2}=$ $6 / \beta$ and the other two terms represent the fermionic contributions. The WilsonDirac operator is

$$
a\left(D_{\mathrm{w}}+\delta D+m_{0}\right)=\frac{1}{2 \kappa} M, \quad \kappa=\left(8+2 a m_{0}\right)^{-1},
$$

where $\delta D$ is the correction for $\mathrm{O}(a)$ improvement (clover term). We use even-odd preconditioning [6] and the Wilson-Dirac operator assumes the block form

$$
M=\left(\begin{array}{cc}
1+T_{e e} & M_{e o} \\
M_{o e} & 1+T_{o o}
\end{array}\right),
$$

where the subscripts $e(o)$ refer to the even (odd) sites of the lattice. The boundary values of the quark fields are set to zero and in this case the $\mathrm{O}(a)$ improvement terms proportional to $\left(\tilde{c}_{t}-1\right)$ do not contribute to the off-diagonal blocks [7]. The operators $T_{e e}$ and $T_{o o}$ are diagonal in space-time and represent the $\mathrm{O}(a)$ corrections. The Hermitian operator $\gamma_{5} M$ can be written as the product

$$
\left(\begin{array}{cc}
\gamma_{5}\left(1+T_{e e}\right) & 0 \\
\gamma_{5} M_{o e} & 1
\end{array}\right) \times\left(\begin{array}{cc}
1 & \left(1+T_{e e}\right)^{-1} M_{e o} \\
0 & \gamma_{5}\left\{1+T_{o o}-M_{o e}\left(1+T_{e e}\right)^{-1} M_{e o}\right\}
\end{array}\right) .
$$

We define the following Hermitian operators

$$
\begin{array}{cl}
Q=c_{0} \gamma_{5} M, & c_{0}=(1+8 \kappa)^{-1}, \\
\hat{Q}=\tilde{c}_{0} \gamma_{5}\left\{1+T_{o o}-M_{o e}\left(1+T_{e e}\right)^{-1} M_{e o}\right\}, & \tilde{c}_{0}=\left(1+64 \kappa^{2}\right)^{-1} .
\end{array}
$$

The fermionic contribution to the partition function can then be written as

$$
\operatorname{det}\left(Q^{2}\right) \propto \operatorname{det}\left(1+T_{e e}\right)^{2} \operatorname{det} \hat{Q}^{2} .
$$

From eq. (2.8) we get the contributions to $S_{\text {eff }}$ in eq. (2.2)

$$
\begin{aligned}
S_{\mathrm{det}} & =-2 \operatorname{tr} \ln \left(1+T_{e e}\right) \\
\operatorname{det}\left(\hat{Q}^{2}\right) & \propto \int \mathrm{D}[\phi] \exp \left(-S_{\mathrm{PF}}\right), \quad S_{\mathrm{PF}}=\phi^{\dagger} \hat{Q}^{-2} \phi,
\end{aligned}
$$

in terms of one dynamical complex pseudo-fermion field $\phi$.

In $[1,2]$ a modified effective pseudo-fermionic action has been proposed. By using the identity $\hat{Q}=\tilde{Q} \tilde{Q}^{-1} \hat{Q}$ for some arbitrary invertible matrix $\tilde{Q}$, we can compute $\operatorname{det}\left(\hat{Q}^{2}\right)$ by using two instead of one pseudo-fermion fields

$$
\begin{aligned}
\operatorname{det}\left(\hat{Q}^{2}\right) & \propto \int\left(\prod_{i=1}^{n_{\mathrm{pf}}} \mathrm{D}\left[\phi_{i}\right]\right) \exp \left(-\sum_{i=1}^{n_{\mathrm{pf}}} S_{\mathrm{F}_{i}}\right), \quad n_{\mathrm{pf}}=2 \\
S_{\mathrm{F}_{1}} & =\phi_{1}^{\dagger}\left(\tilde{Q} \tilde{Q}^{\dagger}\right)^{-1} \phi_{1} \\
S_{\mathrm{F}_{2}} & =\phi_{2}^{\dagger}\left(\hat{Q}^{-1} \tilde{Q} \tilde{Q}^{\dagger} \hat{Q}^{-1}\right) \phi_{2} .
\end{aligned}
$$


In this work we choose

$$
\tilde{Q}=\hat{Q}-i \rho
$$

where $\rho$ is a real number. We can then cast eq. (2.12) and eq. (2.13) into the form

$$
S_{\mathrm{F}_{i}}=\phi_{i}^{\dagger}\left[\sigma_{i}^{2}+\left(\hat{Q}^{2}+\rho_{i}^{2}\right)^{-1}\right] \phi_{i}, \quad i=1,2,
$$

where the field $\phi_{2}$ has been rescaled and

$$
\begin{aligned}
\sigma_{1}=0, & \rho_{1}=\rho, \\
\sigma_{2}=\frac{1}{\rho}, & \rho_{2}=0 .
\end{aligned}
$$

In the Hamiltonian eq. (2.1) the effective action is now given by

$$
S_{\mathrm{PF}}=\sum_{i=1}^{2} S_{\mathrm{F}_{i}}\left(U, \phi_{i}, \phi_{i}^{\dagger}\right) .
$$

We determine the parameter $\rho$ in eq. (2.14) by requiring that the sum $K$ of the condition numbers of the operators appearing in the actions $S_{\mathrm{F}_{1}}$ and $S_{\mathrm{F}_{2}}$ is minimal. If we use the notation $\lambda_{\min } \equiv \lambda_{\min }\left(\hat{Q}^{2}\right), \lambda_{\max } \equiv \lambda_{\max }\left(\hat{Q}^{2}\right)$ for the lowest respectively highest eigenvalue of $\hat{Q}^{2}$ we get

$$
K=y(\rho)+\frac{k}{y(\rho)}, \quad y(\rho)=\frac{\lambda_{\max }+\rho^{2}}{\lambda_{\min }+\rho^{2}},
$$

where

$$
k=\frac{\lambda_{\max }}{\lambda_{\min }}
$$

is the condition number of $\hat{Q}^{2}$. The minimal $K$ is reached for $y(\rho)=\sqrt{k}$. The condition numbers of the operators in $S_{\mathrm{F}_{1}}$ and $S_{\mathrm{F}_{2}}$ are then both equal to $\sqrt{k}$. Solving for $\rho$ we obtain $\rho=\left(\lambda_{\min } \lambda_{\max }\right)^{1 / 4}$. We set $^{1}$

$$
\rho=\left(\left\langle\lambda_{\min }\right\rangle\left\langle\lambda_{\max }\right\rangle\right)^{1 / 4}
$$

where we denote by $\langle\cdot\rangle$ the expectation value computed in a Monte Carlo simulation. Of practical advantage is the following relation which holds at tree level in the Schrödinger functional [8]

$$
k \propto\left(\frac{T}{a}\right)^{2},
$$

\footnotetext{
${ }^{1}$ In practice $\lambda_{\max }$ fluctuates negligibly compared to $\lambda_{\min }$.
} 
where $T$ is the temporal extension of the box. Qualitatively the same behavior (at the critical mass) is expected for

$$
\langle k\rangle=\left\langle\frac{\lambda_{\max }}{\lambda_{\min }}\right\rangle \propto\left(\frac{T}{a}\right)^{2}
$$

in the continuum limit at fixed renormalized coupling $\bar{g}^{2}(L)=u$, where $L$ is the spatial extension of the box. This means that $\rho$ as defined in eq. (2.21) should be scaled with the lattice size $T / a$ approximately like $\sqrt{a / T}$.

The algorithm, which we use in our simulations of eq. (2.1) and eq. (2.18) is Hybrid Monte Carlo [9,10] and consists of the following 4 steps:

1. The momenta $\pi(x, \mu)$ are generated from the Gaussian distribution of their components in a basis of the Lie Algebra of $\mathrm{SU}(3)$.

2. The two pseudo-fermion fields $\phi_{1}$ and $\phi_{2}$ are generated by global heatbath according to the probability distribution

$$
P\left(\phi_{i}\right) \propto \exp \left\{-\phi_{i}^{\dagger}\left[\sigma_{i}^{2}+\left(\hat{Q}^{2}+\rho_{i}^{2}\right)^{-1}\right] \phi_{i}\right\} \propto \exp \left\{-R^{\dagger} R\right\} .
$$

This is done as usual by generating a complex Gaussian random vector $R$. For $\sigma_{i} \neq 0$ we use

$$
\left(\hat{Q}^{2}+\rho_{i}^{2}+\sigma_{i}^{-2}\right) \phi_{i}=\sigma_{i}^{-1}\left(\hat{Q}-i \sqrt{\rho_{i}^{2}+\sigma_{i}^{-2}}\right)\left(\hat{Q}-i \rho_{i}\right) R
$$

If $\sigma_{i}=0$ we use

$$
\phi_{i}=\left(\hat{Q}-i \rho_{i}\right) R .
$$

3. The gauge links $U(x, \mu)$ and the momenta $\pi(x, \mu)$ are evolved along a trajectory of length $\tau$ by integrating the molecular dynamics equations of motion with stepsize $\delta \tau$. This can be done in full analogy with [11]. In the equations of motion for the momenta we need the variation $\delta S_{\mathrm{F}_{i}}$ of the pseudo-fermion actions under an infinitesimal change of the gauge link $\delta U(x, \mu)$. With the help of the vectors

$$
\begin{aligned}
X_{i} & =\left(\hat{Q}^{2}+\rho_{i}^{2}\right)^{-1} \phi_{i}, \\
Y_{i} & =\left(\hat{Q}+i \rho_{i}\right) X_{i},
\end{aligned}
$$

defined on the odd sites of the lattice we construct over the full lattice the vectors

$$
\bar{X}_{i}=\left(\begin{array}{c}
-\left(1+\delta M_{e e}\right)^{-1} M_{e o} X_{i} \\
X_{i}
\end{array}\right), \bar{Y}_{i}=\left(\begin{array}{c}
-\left(1+\delta M_{e e}\right)^{-1} M_{e o} Y_{i} \\
Y_{i}
\end{array}\right),
$$

which we use to write

$$
\delta S_{\mathrm{F}_{i}}=-\frac{\tilde{c}_{0}}{c_{0}}\left(\bar{Y}_{i}^{\dagger} \delta Q \bar{X}_{i}+\bar{X}_{i}^{\dagger} \delta Q \bar{Y}_{i}\right) .
$$


The variation of the two pseudo-fermion actions can be expressed in terms of the variation of the operator $\delta Q$, in the same way as for the standard version with one pseudo-fermion field.

As integration scheme we use an improved Sexton-Weingarten [12] integrator with different time scales for the gauge part and for the pseudo-fermion part of the force which governs the evolution of the momenta. Our integration scheme is the same as the one used in [13] and partially removes the step-size errors $\mathrm{O}\left((\delta \tau)^{3}\right)$ in one integration step.

4. Metropolis accept/reject step: the new configuration $\left\{\pi^{\prime}, U^{\prime}\right\}$ is accepted with probability

$$
\min \{1, \exp (-\Delta H)\}
$$

where

$$
\Delta H=H\left(\pi^{\prime}, U^{\prime}, \phi_{i}\right)-H\left(\pi, U, \phi_{i}\right) .
$$

We monitor $\exp (-\Delta H)$ to check for the correctness of the algorithm, in particular this quantity has proved to be sensitive to the required numerical reversibility in the integration of the equations of motion. For Metropolis-like algorithms it is possible to prove the general property

$$
\langle\exp (-\Delta H)\rangle=1 .
$$

We emphasize that this expectation value is sensitive to all proposed field configurations not only to the accepted ones.

\section{$3 \quad$ Numerical results}

We present a numerical study of simulations of two degenerate massless quarks in the $\mathrm{O}(a)$ on-shell improved Schrödinger functional using the algorithm presented in the previous section. For the lattice temporal and spatial extensions, the background gauge field and the parameter $\theta$ controlling the spatial boundary conditions of the fermion fields we set respectively

$$
T=L, \quad C=C^{\prime}=0, \quad \theta=0.5 .
$$

(see [5] for more detailed definitions of these quantities). This choice is motivated by the fact that our algorithmic study is part of large scale simulations performed by the ALPHA collaboration to determine the running of the renormalized quark mass in two flavor QCD [4]. The massless theory is defined in the bare parameter space along the line $\kappa=\kappa_{c}(\beta)$ where the PCAC mass

$$
m=\left.\frac{\frac{1}{2}\left(\partial_{0}^{*}+\partial_{0}\right) f_{\mathrm{A}}\left(x_{0}\right)+c_{\mathrm{A}} a \partial_{0}^{*} \partial_{0} f_{\mathrm{P}}\left(x_{0}\right)}{2 f_{\mathrm{P}}\left(x_{0}\right)}\right|_{x_{0}=T / 2}
$$


vanishes $^{2}$. Here, $\partial_{0}$ and $\partial_{0}^{*}$ are the forward and backward lattice derivatives, respectively, and $c_{\mathrm{A}}$ denotes the coefficient multiplying the $\mathrm{O}(a)$ improvement term in the improved axial current. For the definitions of the correlation functions $f_{\mathrm{A}}$ and $f_{\mathrm{P}}$ we follow [14]. We stress the fact that simulations along the massless line are possible since the Schrödinger functional has a natural infrared cut-off proportional to $1 / T^{2}$ in the spectrum of the Dirac operator squared [8]. If the fluctuations of the gauge field are not too large this cut-off avoids the occurrence of small eigenvalues and thus makes simulations of the massless theory possible in not too large physical volumes.

In the Schrödinger functional the renormalized coupling $u=\bar{g}^{2}(L)$ is presumably a monotonically growing function of $L$ [15-17]. Keeping the physical size $L$ constant is equivalent to holding the renormalized coupling fixed. We perform simulations approximately at couplings

$$
u=1.0,1.1,1.2,1.3,1.5,1.8,2.0,2.5,3.3,5.7,
$$

and for each coupling at various lattice sizes $L / a$ corresponding to different lattice resolutions. A summary of the bare parameters and the formulae used for the $\mathrm{O}(a)$ improvement coefficients as functions of the bare gauge coupling can be found in Appendix A. Moreover we did some exploratory simulations at $\beta=5.2$ and $\kappa=0.1355$ with lattice sizes $L / a=6,8$ and 12 . The corresponding renormalized coupling is presently only known for $L / a=6$ to be $u=4.7$. The value $\kappa=0.1355$ is the same as for the lightest quark mass used in the large volume simulations of Refs. $[18,19]$. As long as a low energy reference scale is not determined, we cannot yet associate the $u$ values with physical units of the box size $L$. We use the renormalized couplings to "label" our simulations. Indicatively the couplings in eq. (3.3) correspond to the range $10^{-2} \mathrm{fm} \ldots 1 \mathrm{fm}$.

In the simulations we report on here the quantity we are primarily interested in is the renormalization constant of the pseudoscalar density $Z_{\mathrm{P}}$. For a definition of $Z_{\mathrm{P}}$ see [14]. In the massless renormalization scheme that we employ, $Z_{\mathrm{P}}$ depends on the size of the system and on the lattice resolution. We are interested in $Z_{\mathrm{P}}$ since its running with the renormalization scale $\mu=1 / L$ determines the running of the renormalized quark mass $\bar{m}(\mu)$ :

$$
\frac{\bar{m}(\mu)}{\bar{m}(\mu / 2)}=\left.\lim _{a / L \rightarrow 0} \frac{Z_{\mathrm{P}}\left(g_{0}, 2 L / a\right)}{Z_{\mathrm{P}}\left(g_{0}, L / a\right)}\right|_{\bar{g}^{2}(L)=u} .
$$

\footnotetext{
${ }^{2}$ Note that many choices of matrix elements of the PCAC relation yield masses which agree up to $\mathrm{O}\left(a^{2}\right)$ effects. The mass definitions can differ for instance in the choice of the parameters eq. (3.1), of $x_{0}$ eq. (3.2) or of the boundary states.
} 
As a measure of the CPU cost of our simulations we take the integrated autocorrelation time of $Z_{\mathrm{P}}$ per lattice point

$$
\tau_{\text {int }}\left(Z_{\mathrm{P}}\right)[\mathrm{sec}]=\tau_{\text {int }}\left(Z_{\mathrm{P}}\right)[\mathrm{MD}] \times t_{\mathrm{MD}} \times\left(\frac{a}{T}\right)\left(\frac{a}{L}\right)^{3} .
$$

We denote by $\tau_{\text {int }}\left(Z_{\mathrm{P}}\right)$ [MD] the integrated autocorrelation time [20] in units of molecular dynamics (MD) time. In eq. (3.5) $t_{\mathrm{MD}}$ are the CPU seconds needed on one APEmille crate for one update move of one unit of MD time. Besides being machine-dependent $t_{\mathrm{MD}}$ depends on the algorithm and on the step-size $\delta \tau$. One APEmille crate consists of 128 nodes whose peak performance adds up to 68 GFlops. A trivial volume factor is divided out in eq. (3.5). One call of the operator $\hat{Q}$ on APEmille costs roughly $12 \mu$ sec per lattice point (depending on communication). By dividing the value of $\tau_{\text {int }}\left(Z_{\mathrm{P}}\right)$ [sec] by $12 \mu \mathrm{sec}$ we get the integrated autocorrelation time expressed in the equivalent number of applications of $\hat{Q}$.

\subsection{Numerical precision}

We carry out the numerical simulations in single precision arithmetics ${ }^{3}$. In this section we study effects of the numerical precision of our simulations using $n_{\mathrm{pf}}=2$ pseudo-fermion fields by changing some of the algorithmic parameters.

During the integration of the molecular dynamics equations of motion the inversions of the operators $\hat{Q}^{2}+\rho^{2}$ and $\hat{Q}^{2}$ required in eq. (2.27) are performed with the conjugate gradient $(\mathrm{CG})$ iteration algorithm. We use as a stopping criterion the relative residue which is defined as the square of the ratio between the norm of the residue vector and the norm of the present solution vector. The iteration stops whenever this number reaches the requested accuracy $\varepsilon^{2}$. The starting vector for each inversion along the trajectory is chosen to be zero. We first investigated the possibility of increasing the relative residue compared to the single precision value $\varepsilon^{2}=10^{-13}$, thereby saving CPU time. We emphasize that detailed balance holds for all values of $\varepsilon^{2}$. In Table 1 we compare the results for the choice $\varepsilon^{2}=10^{-10}$. We observe a $2 \sigma$ deviation in $Z_{\mathrm{P}}$ and an almost $5 \sigma$ deviation of $\langle\exp (-\Delta H)\rangle$ from 1 .

We have recently learned that our definition of the relative residue $\varepsilon^{2}$ unfortunately differs from the one commonly used in the numerical mathematics literature. There $\varepsilon^{2}$ is defined as the square of the ratio between the norm of the residue vector and the norm of the source vector, which is independent of the normalization of the operator inverted on the source vector. We checked the

\footnotetext{
${ }^{3}$ Global sums are performed in double precision.
} 


\begin{tabular}{|ccccccc|}
\hline$\tau$ & $\varepsilon^{2}$ & $Z_{\mathrm{P}}$ & $\tau_{\text {int }}\left(Z_{\mathrm{P}}\right)[\mathrm{MD}]$ & plaq. & $a m$ & $\langle\exp (-\Delta H)\rangle$ \\
\hline \hline 1 & $10^{-13}$ & $0.6236(17)$ & $22(8)$ & $0.68756(1)$ & $-0.00122(3)$ & $0.989(7)$ \\
1 & $10^{-10}$ & $0.6288(22)$ & $32(12)$ & $0.68756(1)$ & $-0.00118(3)$ & $1.038(8)$ \\
\hline
\end{tabular}

Table 1: Comparison of simulation results for $n_{\mathrm{pf}}=2$ with the relative residue set to the full single precision value $\varepsilon^{2}=10^{-13}$ and to $\varepsilon^{2}=10^{-10}$. Results from $L / a=24$ lattices at coupling 2.5 .

difference between these two definitions of $\varepsilon^{2}$ in the simulations of Table 1 . When $\varepsilon^{2}$ normalized using the solution vector reaches the requested precision $10^{-13}$ or $10^{-10}$, the values of $\varepsilon^{2}$ normalized using the source vector are about 10 times larger. To reach $\varepsilon^{2}=10^{-13}$ defined with the latter normalization it requires an increase of $25 \%$ in CG iterations.

Relaxing the relative residue in the CG inversion leads to a systematic violation of eq. (2.33). Since this possibly indicates the violation of reversibility in the integration of the equations of motion we investigate this issue in more detail in the next section.

\subsection{Reversibility}

The stability of the integration of molecular dynamics equations of motion in connection with Hybrid Monte Carlo algorithms for lattice QCD has been investigated in the literature [21-23]. We report here on our experiences looking at the reversibility of the integration of the equations of motion with $n_{\mathrm{pf}}=2$ pseudofermion fields. We compare how quantities which we monitor to check reversibility behave when the lattice spacing or the trajectory length are changed.

\subsubsection{Diagnostics}

We perform reversibility tests by integrating over one trajectory of length $\tau$ "forward" in molecular dynamics time, reversing at the end of the trajectory the signs of the momenta and integrating "backward" in time over one trajectory of the same length $\tau$. We obtain a cycle:

$$
\{\pi, U\} \stackrel{\tau}{\longrightarrow}\left\{\pi^{\prime}, U^{\prime}\right\} \rightarrow\left\{-\pi^{\prime}, U^{\prime}\right\} \stackrel{\tau}{\longrightarrow}\left\{\pi^{\prime \prime}, U^{\prime \prime}\right\}
$$

Reversibility means that the final configuration $\left\{\pi^{\prime \prime}, U^{\prime \prime}\right\}$ is equal to the starting one $\{\pi, U\}$. We quantify irreversibility by defining the quantities

$$
\mid \Delta H(\text { cycle })|=| H\left(\pi, U, \phi_{i}\right)-H\left(\pi^{\prime \prime}, U^{\prime \prime}, \phi_{i}\right) \mid
$$



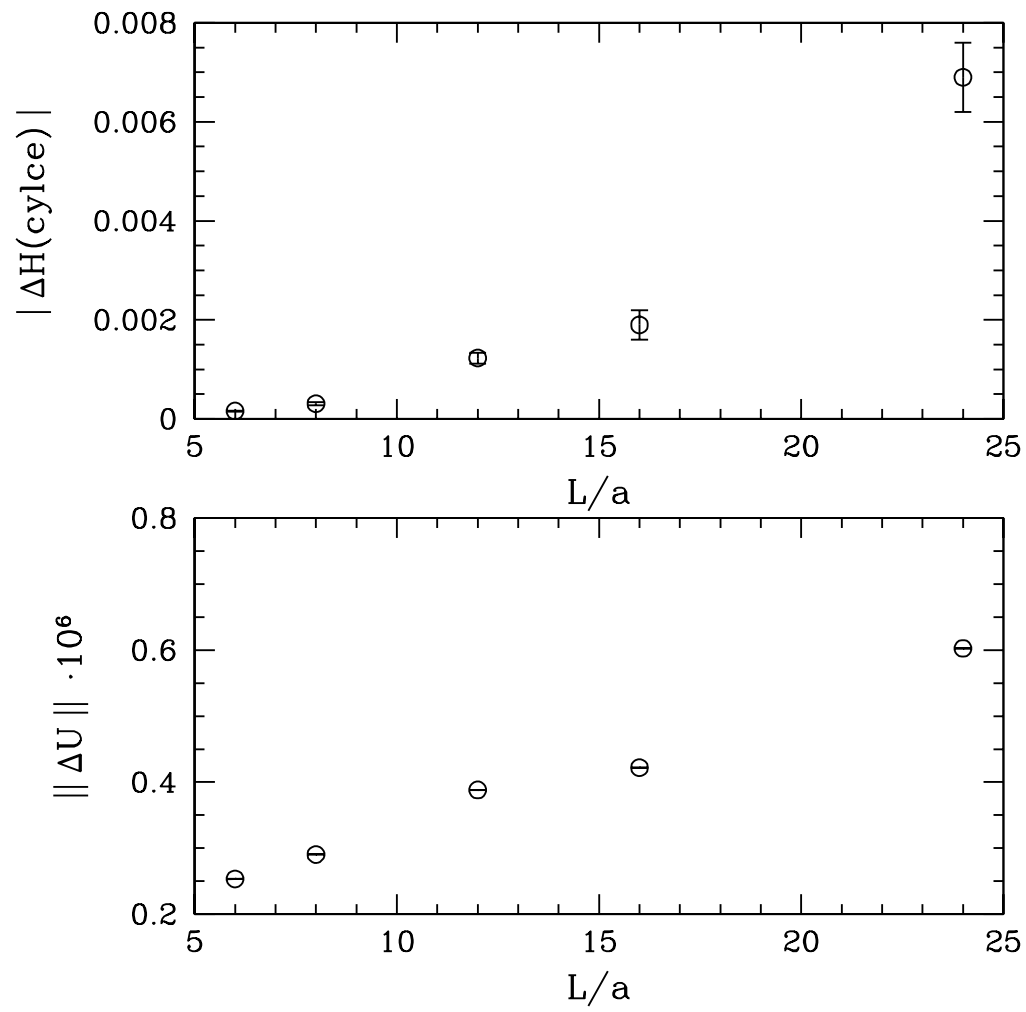

Figure 1: Reversibility violations as functions of the lattice spacing for $n_{\mathrm{pf}}=2$. The renormalized coupling is 2.5. The trajectories used to compute the quantities in eq. (3.7) and eq. (3.8) have length $\tau=1$.

$$
\|\Delta U\|^{2}=\frac{1}{36 L^{4}} \sum_{x, \mu, c, c^{\prime}}\left|U(x, \mu)_{c c^{\prime}}-U^{\prime \prime}(x, \mu)_{c c^{\prime}}\right|^{2}
$$

where $c, c^{\prime}$ are the color indices. The step-size is chosen as in our production runs to keep the acceptance close to $80 \%$ with $\tau=1$ and the relative residue is set to $\varepsilon^{2}=10^{-13}$.

In Fig. 1 we show the irreversibility as a function of the lattice spacing. We plot averages of the quantities eq. (3.7) and eq. (3.8) computed on several configurations, which are well spaced in the Monte Carlo history of our production runs to avoid autocorrelations. The trajectories for these reversibility tests have all length $\tau=1$, the step-size ranges from $1 / 5$ on $L / a=6$ to $1 / 16$ on $L / a=24$ lattices. The renormalized coupling has the value 2.5 , so increasing $L / a$ decreases the lattice spacing by the same factor.

In Fig. 2 we show the irreversibility as a function of the trajectory length $\tau$ 

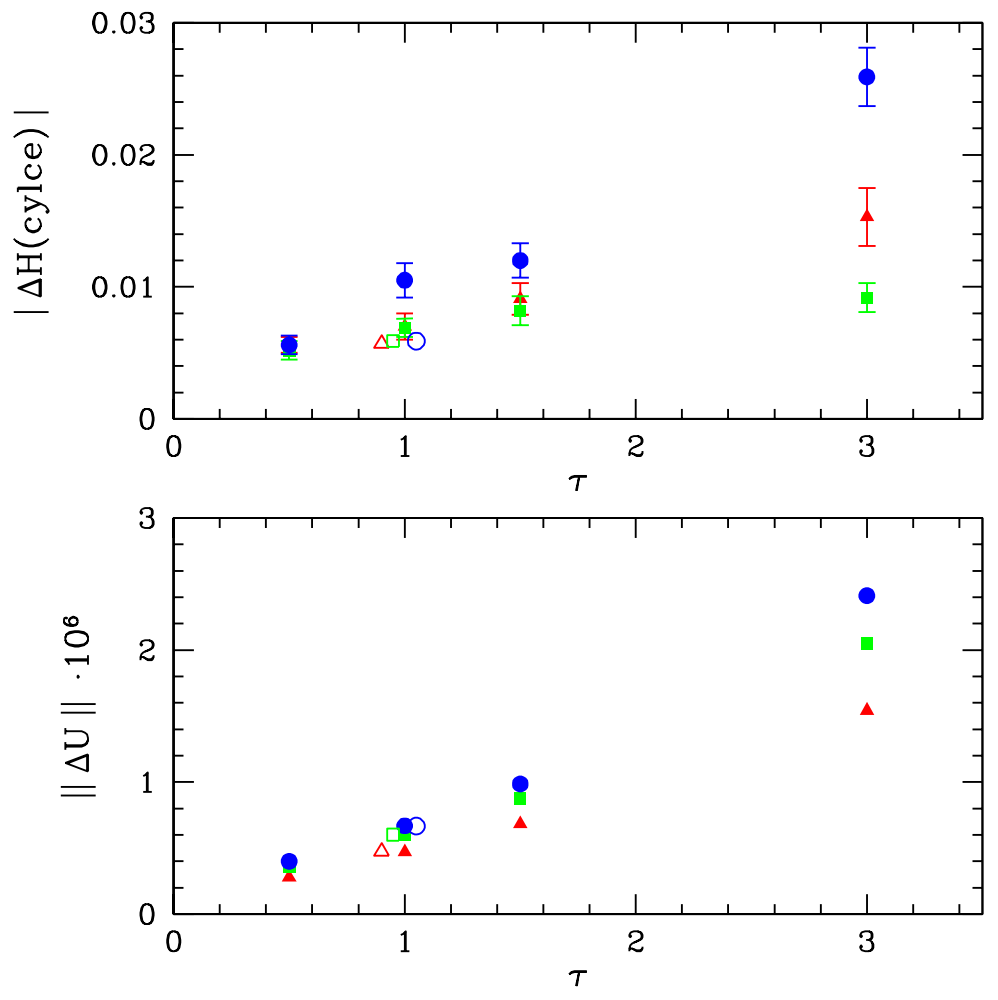

Figure 2: Reversibility violations as functions of the trajectory length on $L / a=24$ lattices for $n_{\mathrm{pf}}=2$. The renormalized couplings are 1.1 (triangles), 2.5 (squares) and 5.7 (circles). The open symbols for $\tau=1$, slightly displaced for clarity, are obtained by switching off the fermions during the MD evolution.

used to compute the quantities eq. (3.7) and eq. (3.8). Varying $\tau$ for fixed stepsize gives us a handle to artificially change the amount of reversibility violations. We plot results for three different couplings 1.1 (triangles), 2.5 (squares) and 5.7 (circles) on $L / a=24$ lattices. The step-sizes are $1 / 12,1 / 16$ and $1 / 18$ respectively. The quantities in eq. (3.7) and eq. (3.8) are averaged over 30 well spaced configurations taken from our production runs. The relative increase of $\mid \Delta H$ (cycle) $\mid$ and $\|\Delta U\|$ with increasing $\tau$ is of comparable magnitude. In general the irreversibility is slightly larger for larger coupling.

For $\tau=1$ we also considered the case when the fermions are switched off in the integration of the equations of motion. This corresponds to the open symbols in Fig. 2, which are slightly displaced horizontally to distinguish them. We conclude that the reversibility violations coming from the gauge part of the force which 
governs the evolution of the momenta are not negligible with respect to the ones coming from the pseudo-fermion part.

\subsubsection{Influence on observables}

To check that we can safely simulate a trajectory of length $\tau=1$ with the relative residue in the $\mathrm{CG}$ iterations set to $\varepsilon^{2}=10^{-13}$, we repeated one simulation on $L / a=24$ lattices at coupling 1.1 with trajectory length $\tau=0.5$, keeping the other parameters unchanged. As it is shown in Fig. 2 the reversibility violations for $\tau=0.5$ are smaller than for $\tau=1$ and so we consider simulations with $\tau=0.5$ as a safe reference for the correct mean values of observables we are interested in.

The results are shown in Table 2 and confirm our expectation. In addition we do not see a difference in efficiency between $\tau=0.5$ and $\tau=1$ within our errors on $\tau_{\text {int }}\left(Z_{\mathrm{P}}\right)$.

\begin{tabular}{|ccccccc|}
\hline$\tau$ & $\varepsilon^{2}$ & $Z_{\mathrm{P}}$ & $\tau_{\text {int }}\left(Z_{\mathrm{P}}\right)[\mathrm{MD}]$ & plaq. & $a m$ & $\langle\exp (-\Delta H)\rangle$ \\
\hline \hline 1 & $10^{-13}$ & $0.7828(13)$ & $26(8)$ & $0.78876(1)$ & $-0.00076(2)$ & $1.012(10)$ \\
0.5 & $10^{-13}$ & $0.7813(13)$ & $24(8)$ & $0.78876(1)$ & $-0.00074(1)$ & $0.998(7)$ \\
\hline
\end{tabular}

Table 2: Comparison of simulation results for $n_{\mathrm{pf}}=2$ using trajectory length $\tau=1$ and $\tau=0.5$. Results stem from $L / a=24$ lattices at coupling 1.1.

From these results and from the ones in Section 3.1 we conclude that it is safe for our purposes to simulate with the relative residue set to $\varepsilon^{2}=10^{-13}$ in the CG inversions and with trajectory length $\tau=1$.

\subsection{Performance}

In this section we compare the performance of our simulations using $n_{\mathrm{pf}}=2$ pseudo-fermion fields with simulations using $n_{\mathrm{pf}}=1$.

\begin{tabular}{|cccccc|}
\hline$n_{\text {pf }}$ & $\delta \tau$ & \# traj. & $P_{\text {acc }}$ & plaq. & $\langle\exp (-\Delta H)\rangle$ \\
\hline \hline 2 & $1 / 18$ & 2744 & $83 \%$ & $0.62725(1)$ & $1.020(9)$ \\
1 & $1 / 36$ & 160 & $49 \%$ & $0.62726(5)$ & $0.81(12)$ \\
1 & $1 / 40$ & 168 & $78 \%$ & $0.62731(5)$ & $0.96(4)$ \\
1 & $1 / 50$ & 168 & $89 \%$ & $0.62732(3)$ & $1.036(27)$ \\
\hline
\end{tabular}

Table 3: Comparison of the step-size between $n_{\mathrm{pf}}=1$ and $n_{\mathrm{pf}}=2$. Results on $L / a=24$ lattices at coupling 5.7. 


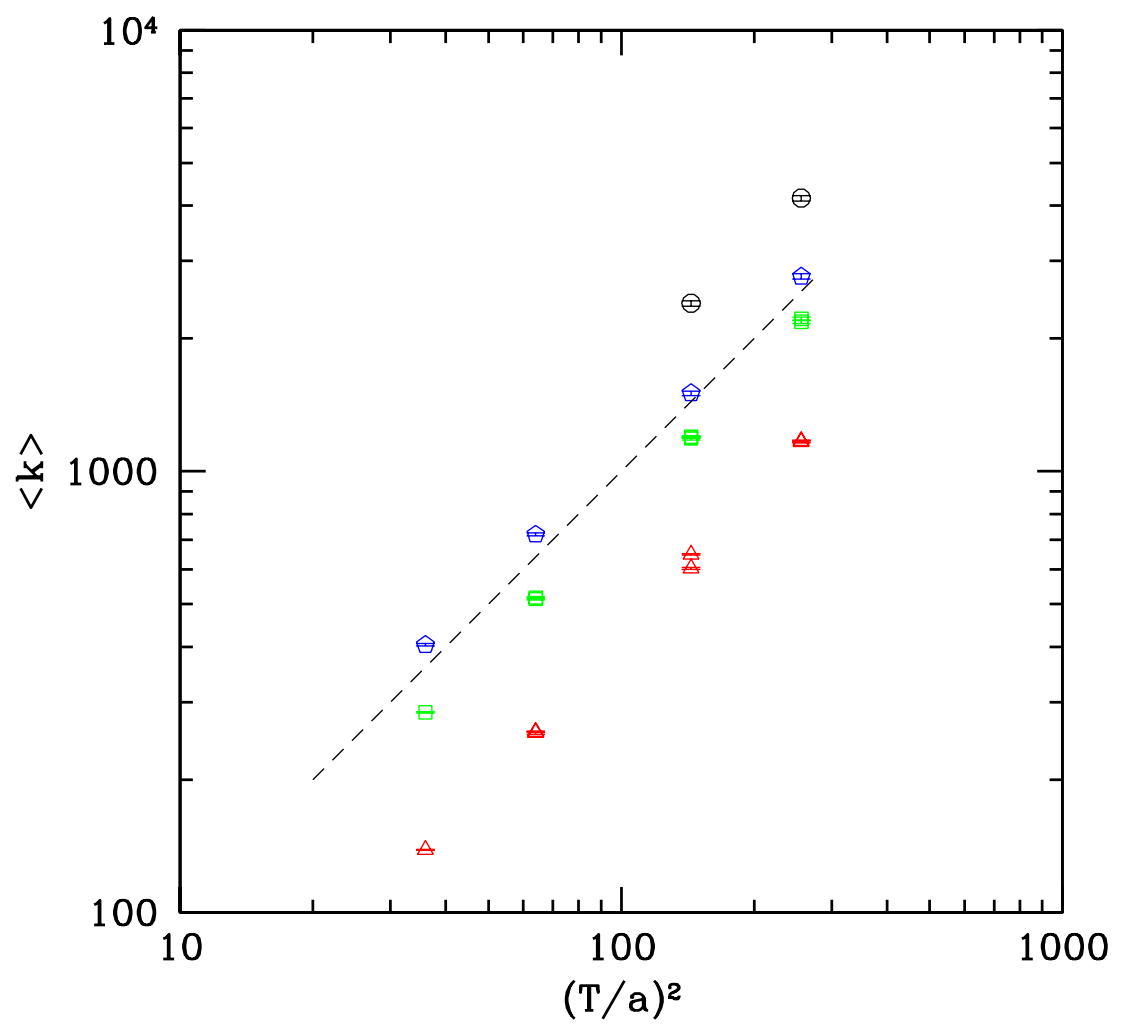

Figure 3: Scaling of the average condition number $\langle k\rangle$ of $\hat{Q}^{2}$ with $(T / a)^{2}$. Data for couplings 1.0 and 1.1 combined (triangles), 2.5 (squares), 3.3 (pentagons) and 5.7 (circles).

First we look at the step-size which is required to get the same acceptance. The acceptances in our simulations typically scatter around 80\%. Following $[24,25]$ we assume that the acceptance probability $P_{\text {acc }}$ depends on the step-size $\delta \tau$ of the improved Sexton-Weingarten integration scheme like

$$
\ln P_{\mathrm{acc}} \approx 1-P_{\mathrm{acc}} \propto(\delta \tau)^{4}
$$

This formula takes into account what are expected to be the dominant discretization errors of the integration. We use eq. (3.9) for a small correction to $\delta \tau$ corresponding to $P_{\text {acc }}=80 \%$ precisely. Table 3 shows results for simulations on $L / a=24$ lattices at coupling 5.7. The acceptance $80 \%$ is achieved with $\delta \tau=1 / 17$ for $n_{\mathrm{pf}}=2$ and $\delta \tau=1 / 41$ for $n_{\mathrm{pf}}=1$. This is more than a factor two difference.

In Fig. 3 we plot our available data for the average condition number $\langle k\rangle$ of the operator $\hat{Q}^{2}$ as function of $(T / a)^{2}$. Data are for couplings 1.0 and 1.1 combined 


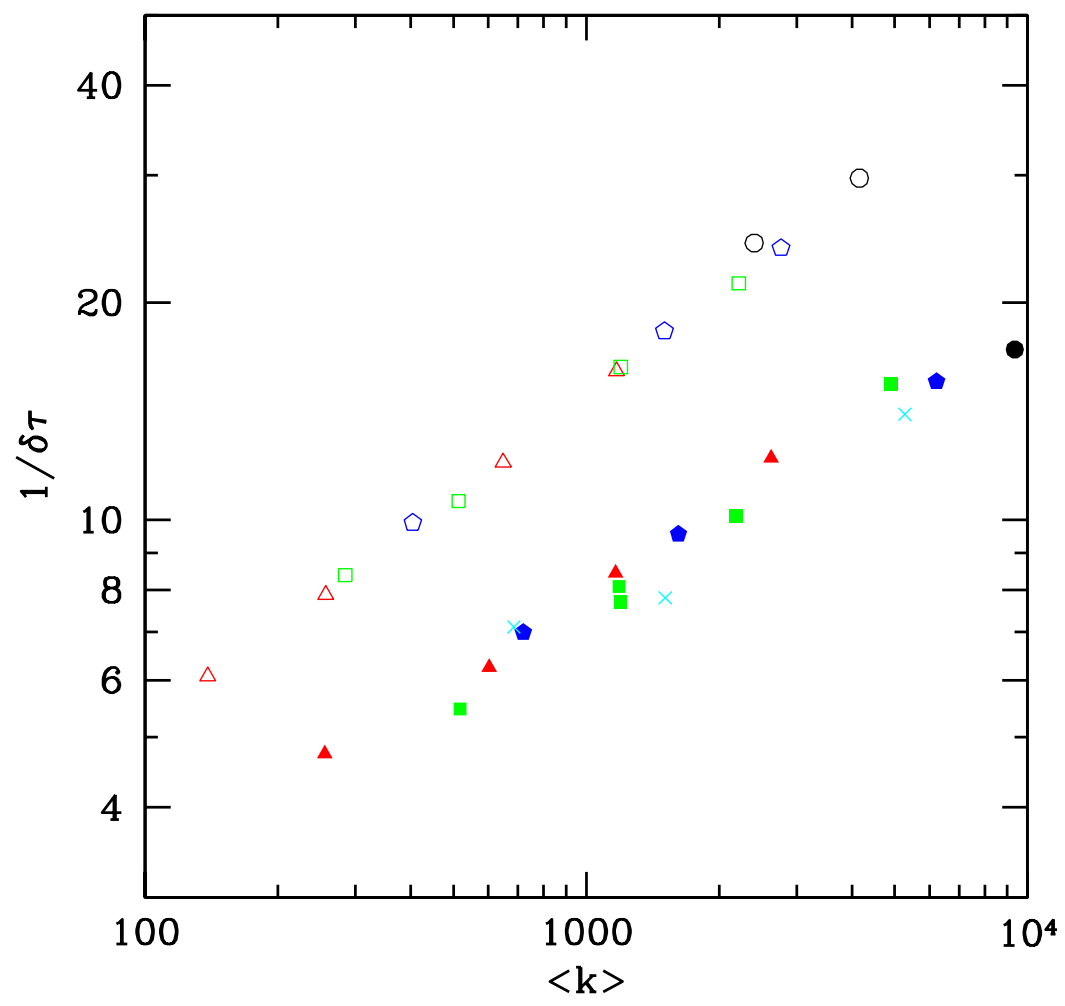

Figure 4: Scaling of the inverse step-size $1 / \delta \tau$ for $80 \%$ acceptance with the average condition number $\langle k\rangle$ of $\hat{Q}^{2}$. Open symbols are for $n_{\mathrm{pf}}=1$, filled symbols and crosses for $n_{\mathrm{pf}}=2$ as explained in the text.

(triangles), 2.5 (squares), 3.3 (pentagons) and 5.7 (circles). They confirm well the relation eq. (2.23) and we use it to extrapolate $\langle k\rangle$ where we do not have measurements of the smallest and highest eigenvalue of $\hat{Q}^{2}$. To guide the eye we draw the dashed line $\langle k\rangle=10(T / a)^{2}$.

In Fig. 4 the inverse step-size (i.e. the number of steps in one trajectory with $\tau=1)$ is plotted against $\langle k\rangle$ in a doubly logarithmic plot. The step-sizes have been corrected according to eq. (3.9) to normalize the acceptance to 0.80. Data are for couplings 1.0 and 1.1 combined (triangles), 2.5 (squares), 3.3 (pentagons) and 5.7 (circles). Open symbols are for $n_{\mathrm{pf}}=1$ and filled symbols for $n_{\mathrm{pf}}=2$. In addition we plot data for $\beta=5.2, \kappa=0.1355, L / a=6,8$ and 12 runs (crosses) with $n_{\mathrm{pf}}=2$. The points both for $n_{\mathrm{pf}}=1$ and $n_{\mathrm{pf}}=2$ fall on almost parallel straight lines showing remarkable scaling for the different couplings used. A parallel displacement in a doubly logarithmic plot corresponds to a change in 


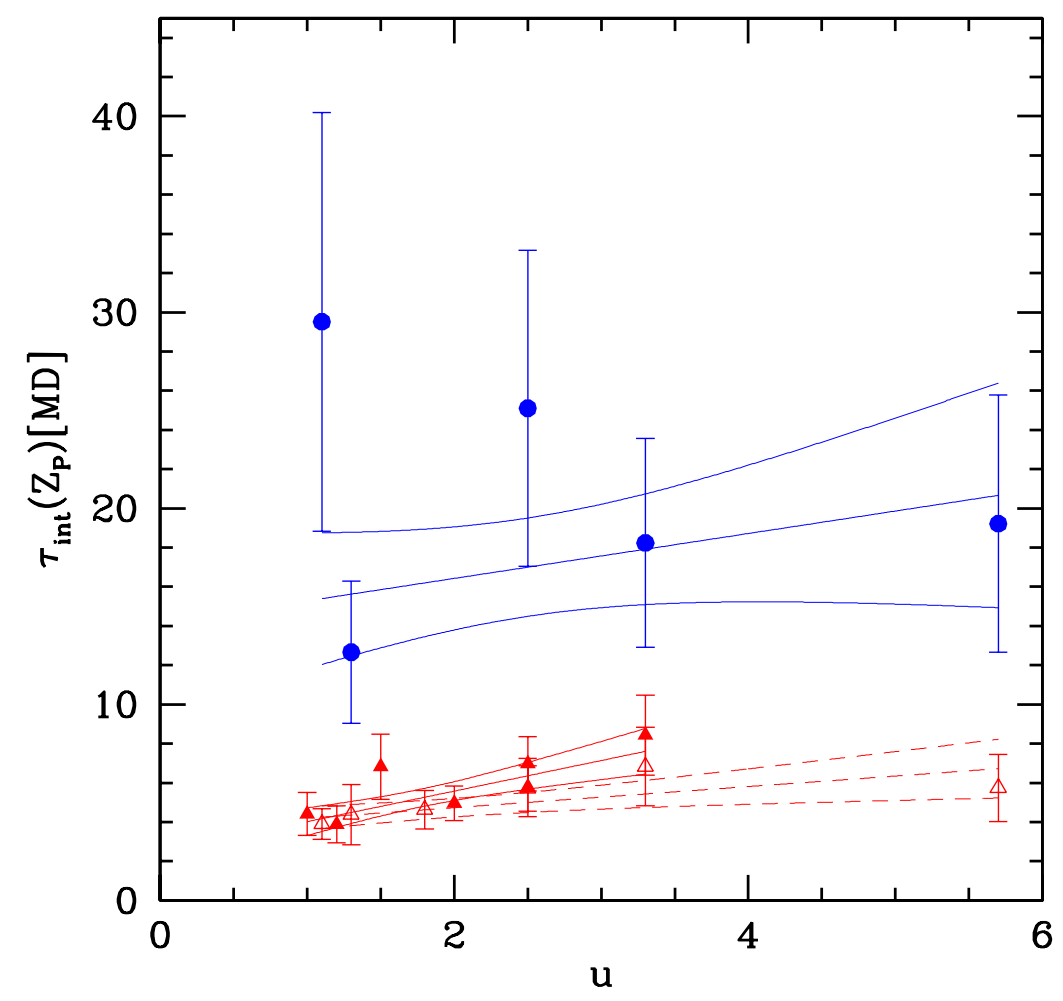

Figure 5: The integrated autocorrelation time $\tau_{\text {int }}\left(Z_{\mathrm{P}}\right)[\mathrm{MD}]$ as function of the renormalized coupling $u$. Data for $L / a=12$ with $n_{\mathrm{pf}}=1$ (open triangles), $L / a=12$ with $n_{\mathrm{pf}}=2$ (filled triangles) and $L / a=24$ with $n_{\mathrm{pf}}=2$ (filled circles) are shown together with linear fits and their $1 \sigma$ error bands.

the pre-factor of the power law relating $1 / \delta \tau$ to $\langle k\rangle$.

In Fig. 5 we plot the integrated autocorrelation time $\tau_{\text {int }}\left(Z_{\mathrm{P}}\right)[\mathrm{MD}]$ as function of the renormalized coupling $u$. A careful analysis of the error of $Z_{\mathrm{P}}$ and $\tau_{\text {int }}\left(Z_{\mathrm{P}}\right)$ has been done using the method described in [20]. Data are for $L / a=12$ (triangles) and $L / a=24$ (circles) lattices, open symbols refer to simulations with $n_{\mathrm{pf}}=1$ and filled symbols to $n_{\mathrm{pf}}=2$. In the coupling range simulated there is no significant dependence on the coupling. We show in the plot the results with error bands of linear fits to the data. Similar fits have been done for the $L / a=16$ lattices not shown in Fig. 5. The fits are used to estimate the error of $Z_{\mathrm{P}}$, so their uncertainty influence the error of this error. We do not observe a significant difference between $n_{\mathrm{pf}}=1$ and $n_{\mathrm{pf}}=2$. This supports the results of [2] and the assumption made in [3]. 


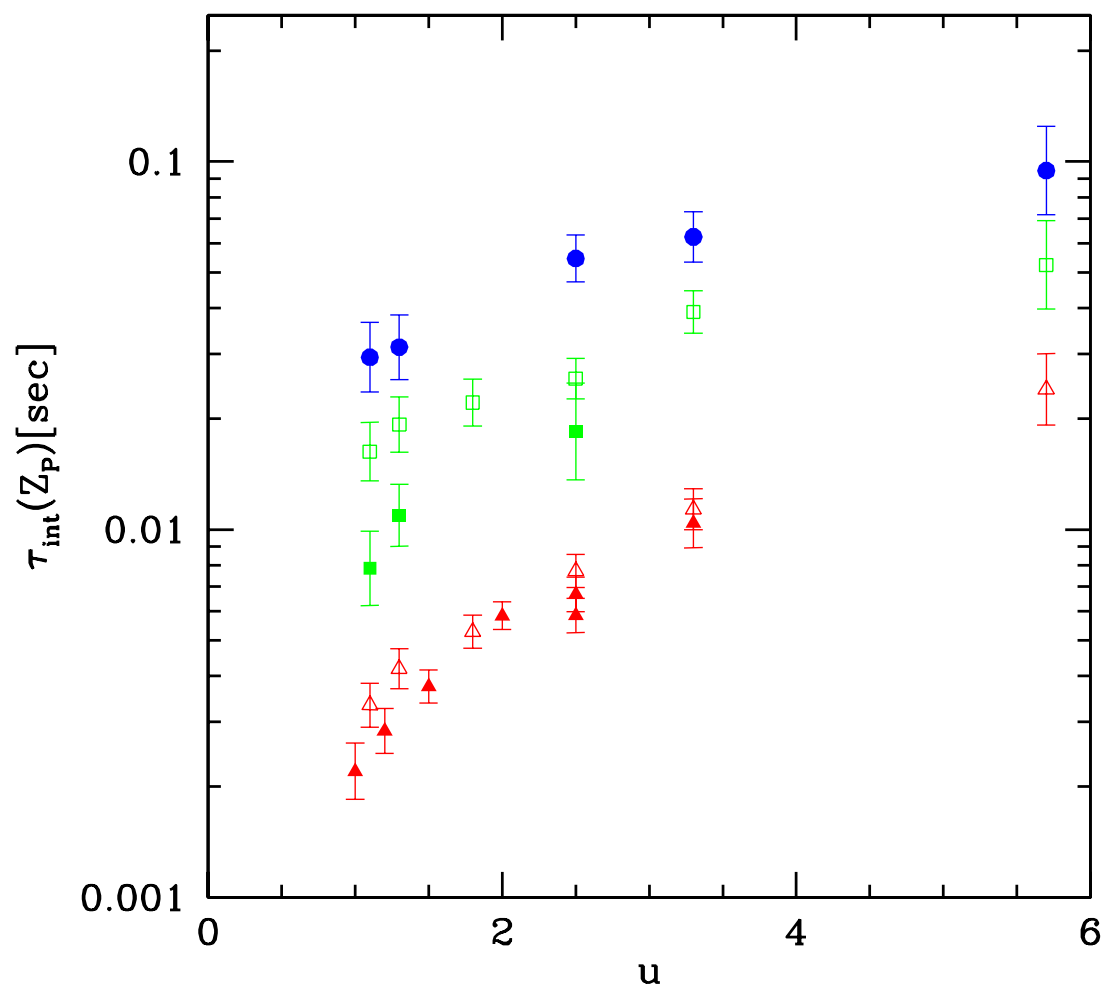

Figure 6: The integrated autocorrelation time $\tau_{\text {int }}\left(Z_{\mathrm{P}}\right)$ [sec] as function of the renormalized coupling $u$. Data for lattices $L / a=12$ (triangles), $L / a=16$ (squares) and $L / a=24$ (circles) are shown, open symbols are for $n_{\mathrm{pf}}=1$ and filled symbols for $n_{\mathrm{pf}}=2$. These data are obtained from eq. (3.5) taking the linear fits for $\tau_{\text {int }}\left(Z_{\mathrm{P}}\right)[\mathrm{MD}]$.

To see the difference between $n_{\mathrm{pf}}=1$ and $n_{\mathrm{pf}}=2$ we plot in Fig. 6 the autocorrelation time $\tau_{\text {int }}\left(Z_{\mathrm{P}}\right)$ [sec] as function of the renormalized coupling $u$. In these units $\tau_{\text {int }}\left(Z_{\mathrm{P}}\right)$ becomes a direct measure of the CPU cost of the algorithms. Data are for $L / a=12$ (triangles), $L / a=16$ (squares) and $L / a=24$ (circles) lattices, open symbols are for $n_{\mathrm{pf}}=1$ and filled symbols for $n_{\mathrm{pf}}=2$. The data shown are obtained from eq. (3.5) taking the values and errors of $\tau_{\text {int }}\left(Z_{\mathrm{P}}\right)[\mathrm{MD}]$, which result from the linear fits in $u$. A systematic difference between $n_{\mathrm{pf}}=1$ and $n_{\mathrm{pf}}=2$ is seen for the $L / a=16$ lattices, the smallest couplings show a reduction of $\tau_{\text {int }}\left(Z_{\mathrm{P}}\right)$ [sec] by about a factor two for $n_{\mathrm{pf}}=2$. We did not run with $n_{\mathrm{pf}}=1$ on $L / a=24$ lattices because, according to our expectations based on the results for the $L / a=16$ lattices, this would be demanding too much CPU time. In Fig. 6 
we see that the value of $\tau_{\text {int }}\left(Z_{\mathrm{P}}\right)$ [sec] for the $L / a=16$ simulation with $n_{\mathrm{pf}}=1$ at the largest coupling 5.7 is within error almost compatible with the value for the $L / a=24$ simulation with $n_{\mathrm{pf}}=2$ at the same coupling. The gain in CPU cost can be larger than a factor two for the $L / a=24$ simulations when using $n_{\mathrm{pf}}=2$ instead of $n_{\mathrm{pf}}=1$, as supported by the results for the step-size in Table 3 .

Based on the step-sizes shown in Fig. 4 and the results for $\tau_{\text {int }}\left(Z_{\mathrm{P}}\right)$ [MD] shown in Fig. 5 we would expect a gain of a factor two in CPU cost for Hybrid Monte Carlo using $n_{\mathrm{pf}}=2$ pseudo-fermion fields compared to $n_{\mathrm{pf}}=1$. There is a computational overhead for using $n_{\mathrm{pf}}=2$ instead of $n_{\mathrm{pf}}=1$ pseudo-fermions, which comes from the inversion of $\hat{Q}^{2}+\rho^{2}$ in addition to the inversion of $\hat{Q}^{2}$, see eq. (2.27). This overhead compared to the number of CG iterations required for the inversion of $\hat{Q}^{2}$ ranges from $20-30 \%$ on $L / a=12$ lattices to $10-15 \%$ on $L / a=24$ lattices and diminishes as the coupling $u$ increases. This explains why the results for the $L / a=12$ lattices shown in Fig. 6 indicate a smaller gain in CPU cost using $n_{\mathrm{pf}}=2$ than for larger lattices.

One should keep in mind that these results have been obtained on small and intermediate physical volumes. The situation in larger volumes might be even better for $n_{\mathrm{pf}}=2$, as our results for coupling 5.7 indicate.

\section{Conclusions}

We investigated the Hybrid Monte Carlo algorithm using two pseudo-fermion fields to simulate two massless flavors in the Schrödinger functional $\mathrm{O}(a)$ improved theory. This study is part of large scale simulations to compute the running of the renormalized quark mass with the physical lattice size $L$ from the running of the renormalization factor $Z_{\mathrm{P}}$ of the pseudoscalar density.

Our results show a gain in CPU cost of a factor two when using two instead of one pseudo-fermions. This gain comes from the larger step-size and is based on the results for the integrated autocorrelation time of $Z_{\mathrm{P}}$. Since our lattices have $L \approx 1 \mathrm{fm}$ at most, it might well be that the gain is larger on larger volumes [26].

Our simulations are performed with single precision arithmetics. We tested the effects of numerical precision and reversibility of the integration of the molecular dynamics equations of motion on observables. We confirm that our simulations satisfy the requirements imposed both on numerical precision and reversibility.

Acknowledgements. We are grateful to M. Hasenbusch for discussions and to M. Lüscher for feedback on the manuscript. We thank NIC/DESY Zeuthen for allocating computer time on the APEmille machine indispensable to this project and the APE group for their professional and constant support. This work is sup- 
ported in part by the European Community's Human Potential Programme, contract HPRN-CT-2000-00145, "Hadron Phenomenology from Lattice QCD" and by the Deutsche Forschungsgemeinschaft in the SFB/TR 09-03, "Computational Particle Physics".

\section{A Simulation parameters}

\begin{tabular}{lll||lll}
\hline$L / a$ & $\beta$ & $\kappa$ & $L / a$ & $\beta$ & $\kappa$ \\
\hline 6 & 9.5000 & 0.1315322 & 6 & 6.6085 & 0.1352600 \\
8 & 9.7341 & 0.1313050 & 8 & 6.8217 & 0.1348910 \\
12 & 10.05755 & 0.1310691 & 12 & 7.09300 & 0.1344320 \\
& & & & & \\
6 & 8.5000 & 0.1325094 & 6 & 6.1330 & 0.1361100 \\
8 & 8.7223 & 0.1322907 & 8 & 6.3229 & 0.1357673 \\
12 & 8.99366 & 0.1319754 & 12 & 6.63164 & 0.1352270 \\
& & & & & \\
6 & 7.5000 & 0.1338150 & 6 & 5.6215 & 0.1366650 \\
8 & 7.7206 & 0.1334970 & 8 & 5.8097 & 0.1366077 \\
12 & 8.02599 & 0.1330633 & 12 & 6.11816 & 0.1361387 \\
\hline
\end{tabular}

Table 4: Summary of simulation parameters.

In Table 4 we list the lattice sizes and bare parameters of our simulations. These are divided in six groups of three sets each. In each group $\beta$ and $\kappa$ have been tuned for the lattice sizes $L / a=6,8,12$ to keep the renormalized coupling $\bar{g}^{2}(L)$ at an approximately fixed value $u$ (see Ref. [15] for a precise definition of $\bar{g}^{2}(L)$ ), in the left column $u=1.0,1.2,1.5$, in the right column $u=2.0,2.5,3.3$ in the order from top to bottom. For each bare parameter set we also simulated the doubled lattice sizes $2 L / a$. The renormalized coupling then takes the values $u=1.1,1.3,1.8$ and $u=2.5,3.3,5.7$ in the same ordering as before.

The $\mathrm{O}(a)$ boundary improvement coefficient of the Wilson plaquette gauge action $c_{\mathrm{t}}$ is set to the perturbative value [27]

$$
c_{\mathrm{t}}=1-0.050718 g_{0}^{2}-0.030 g_{0}^{4} .
$$

For the left part of Table 4 except in the simulations with $\beta=7.7206$ and $\kappa_{c}=$ 0.1334970 we take the 1-loop expression, all the other simulations use the 2-loop expression of $c_{\mathrm{t}}$.

As concerns the $\mathrm{O}(a)$ improvement of the Wilson-Dirac operator, the clover 
coefficient $c_{\mathrm{sw}}$ is computed using the non-perturbative fit-formula given in [28]

$$
c_{\mathrm{sw}}=\frac{1-0.454 g_{0}^{2}-0.175 g_{0}^{4}+0.012 g_{0}^{6}+0.045 g_{0}^{8}}{1-0.720 g_{0}^{2}} .
$$

The coefficient $\tilde{c}_{\mathrm{t}}$ is set to the 1-loop perturbative value [29]

$$
\tilde{c}_{\mathrm{t}}=1-0.01795 g_{0}^{2} .
$$

Finally we use for the $\mathrm{O}(a)$ improvement coefficient of the axial current $c_{\mathrm{A}}$ the 1-loop perturbative value [29]

$$
c_{\mathrm{A}}=-0.007573 g_{0}^{2} .
$$

\section{References}

[1] M. Hasenbusch, Phys. Lett. B519 (2001) 177, hep-lat/0107019.

[2] M. Hasenbusch and K. Jansen, Nucl. Phys. B659 (2003) 299, heplat/0211042.

[3] QCDSF, A. Ali Khan et al., (2003), hep-lat/0303026.

[4] ALPHA, F. Knechtli et al., Nucl. Phys. Proc. Suppl. 119 (2003) 320, heplat/0209025.

[5] M. Lüscher, S. Sint, R. Sommer and P. Weisz, Nucl. Phys. B478 (1996) 365, hep-lat/9605038.

[6] T.A. DeGrand and P. Rossi, Comput. Phys. Commun. 60 (1990) 211.

[7] M. Lüscher and P. Weisz, Nucl. Phys. B479 (1996) 429, hep-lat/9606016.

[8] S. Sint, Nucl. Phys. B421 (1994) 135, hep-lat/9312079.

[9] S. Duane, A.D. Kennedy, B.J. Pendleton and D. Roweth, Phys. Lett. B195 (1987) 216.

[10] S. Gottlieb, W. Liu, D. Toussaint, R.L. Renken and R.L. Sugar, Phys. Rev. D35 (1987) 2531.

[11] K. Jansen and C. Liu, Comput. Phys. Commun. 99 (1997) 221, heplat/9603008. 
[12] J.C. Sexton and D.H. Weingarten, Nucl. Phys. B380 (1992) 665.

[13] K. Jansen and C. Liu, Nucl. Phys. B453 (1995) 375, hep-lat/9506020.

[14] ALPHA, S. Capitani, M. Lüscher, R. Sommer and H. Wittig, Nucl. Phys. B544 (1999) 669, hep-lat/9810063.

[15] ALPHA, A. Bode et al., Phys. Lett. B515 (2001) 49, hep-lat/0105003.

[16] ALPHA, J. Heitger, H. Simma, R. Sommer and U. Wolff, Nucl. Phys. Proc. Suppl. 106 (2002) 859, hep-lat/0110201.

[17] ALPHA, M. Della Morte et al., Nucl. Phys. Proc. Suppl. 119 (2003) 439, hep-lat/0209023.

[18] UKQCD, C.R. Allton et al., Phys. Rev. D65 (2002) 054502, hep-lat/0107021.

[19] JLQCD, S. Aoki et al., (2002), hep-lat/0212039.

[20] ALPHA, U. Wolff, (2003), hep-lat/0306017.

[21] C. Liu, A. Jaster and K. Jansen, Nucl. Phys. B524 (1998) 603, heplat/9708017.

[22] R. Frezzotti and K. Jansen, Nucl. Phys. B555 (1999) 395, hep-lat/9808011.

[23] UKQCD, B. Joo et al., Phys. Rev. D62 (2000) 114501, hep-lat/0005023.

[24] S. Gupta, A. Irback, F. Karsch and B. Petersson, Phys. Lett. B242 (1990) 437.

[25] T. Takaishi, Comput. Phys. Commun. 133 (2000) 6, hep-lat/9909134.

[26] M. Hasenbusch, Proceedings, XXI. International Symposium on Lattice Field Theory (Tsukuba), 2003.

[27] ALPHA, A. Bode, P. Weisz and U. Wolff, Nucl. Phys. B576 (2000) 517, hep-lat/9911018.

[28] ALPHA, K. Jansen and R. Sommer, Nucl. Phys. B530 (1998) 185, heplat/9803017.

[29] S. Sint and P. Weisz, Nucl. Phys. B502 (1997) 251, hep-lat/9704001. 